Questions vives

\section{Questions Vives}

Recherches en éducation

Vol.10 n'20| 2013

Accéder à l'expérience : enjeux, modalités, effets

\title{
La prise en compte de l'expérience dans un dispositif de formation: Tensions et stratégies organisationnelles
}

Take into account the experience in a training program: tension sources

\section{Marie-Christine Presse}

\section{(2) OpenEdition}

\section{Journals}

Édition électronique

URL : http://journals.openedition.org/questionsvives/1378

DOI : 10.4000/questionsvives.1378

ISSN : $1775-433 \mathrm{X}$

Éditeur

Université Aix-Marseille (AMU)

Édition imprimée

Date de publication : 15 novembre 2013

Pagination : 63-76

ISBN : 978-2-912643-44-5

ISSN : 1635-4079

Référence électronique

Marie-Christine Presse, «La prise en compte de l'expérience dans un dispositif de formation:

Tensions et stratégies organisationnelles », Questions Vives [En ligne], Vol.10 n²0 | 2013, mis en ligne le 16 décembre 2013, consulté le 19 avril 2019. URL : http://journals.openedition.org/ questionsvives/1378; DOI : 10.4000/questionsvives.1378

Ce document a été généré automatiquement le 19 avril 2019.

\section{(†)

Questions Vives est mis à disposition selon les termes de la licence Creative Commons Attribution Pas d'Utilisation Commerciale - Pas de Modification 4.0 International. 


\title{
La prise en compte de l'expérience dans un dispositif de formation: Tensions et stratégies organisationnelles
}

Take into account the experience in a training program: tension sources

\author{
Marie-Christine Presse
}

\section{Introduction}

1 La demande croissante de reconnaissance des expériences professionnelles, renforcée par l'introduction de la Validation des Acquis de l'Expérience (VAE) à l'université, assortie d'une préconisation de développement des partenariats avec les entreprises, le tout dans un contexte de compétitivité économique conduit à la transformation des parcours de formation et de l'accès aux certifications universitaires.

2 Ces transformations ne sont cependant pas sans poser questions. Quels effets peut produire la prise en compte de l'expérience dans un dispositif de formation universitaire quand ce dispositif est pour partie sous le contrôle de l'entreprise?

Différents travaux de recherche effectués sur la VAE depuis 2002 (Presse, 2008) ont permis d'identifier certains des obstacles auxquels sont confrontés les candidats lorsqu'ils cherchent à faire valoir les acquis issus de leurs expériences. Or malgré ces obstacles et les difficultés pour accéder à l'expérience vécue (Djelo-Dialo, Clot, 2003) l'intérêt pour la prise en compte de celle-ci ne faiblit pas. Ainsi différents partenaires (université, entreprise, conseil général, salariés de l'entreprise) se sont ainsi trouvés impliqués dans la conception, la mise en œuvre et le déroulement d'un dispositif de formation prenant en compte cette expérience et conduisant à l'obtention d'une licence. Comme toute offre de formation ce dispositif est à considérer comme étant un ensemble résolument hétérogène, comportant des discours, des institutions, des aménagements architecturaux, des décisions réglementaires, des lois, des mesures administratives, des énoncés 
scientifiques, des propositions philosophiques, morales, philanthropiques, bref : du dit aussi bien que du non-dit (Foucault, 2001).

4 Cette contribution ne cherche donc pas à fournir des éléments sur l'accès à l'expérience comme démarche ou méthode mais à comprendre ce qui se joue entre les différents protagonistes «tous intéressés » par cette expérience et comment se manifestent et les tensions et contradictions entre ceux-ci.

5 En effet cette offre de formation se situe dans un contexte de construction européenne d'une société de l'économie de la connaissance dans une histoire française diachronique mettant en tension expérience et formation Ce contexte diachronique et synchronique, présenté a des répercussions sur l'offre universitaire. Les discours des différents protagonistes font apparaître les tensions et les rapports de force qui se sont instaurés entre les différents niveaux (université, organisme, stagiaires) et plus particulièrement les composantes du dispositif où se jouent ces rapports de force.

6 La méthodologie d'analyse mobilisée pour ce travail de recherche-action s'appuie sur deux règles de méthode essentielles: d'une part celle de la totalité structurée qui implique qu'aucun élément d'une situation ne peut être compris indépendamment du tout dans lequel elle s'insère, et d'autre part celle selon laquelle l'Homme fait son histoire dans des conditions historiquement et dialectiquement déterminées.

7 Les données analysées sont issues des documents, des entretiens conduits auprès des protagonistes et de l'implication dans le dispositif ayant permise une observation participante.

\section{L'enracinement du dispositif de formation}

8 Le dispositif de formation d'adultes, dont il est ici question, mis en place à l'université, permet à des salariés d'un organisme de formation de viser l'obtention d'une licence. La particularité de ce dispositif est d'être à mi-chemin entre la VAE et la formation traditionnelle.

9 Ce dispositif prend appui sur l'expérience des professionnels et leurs acquis, déclarés. Cette offre de formation s'est élaborée à la suite d'une communication sur les possibilités d'usage de la VAE au sein de dispositifs de formations universitaires, présentée par moimême chercheur.

10 Un organisme de formation, représenté par son DRH, a manifesté son intérêt pour l'implication de ses salariés dans une telle démarche. Les attentes énoncées par ce DRH étaient les suivantes : sécuriser les parcours et les emplois des seniors (+ de 45ans) ayant une expérience professionnelle de longue durée mais n'ayant aucun diplôme et leur permettre de valoriser leurs expérience

11 Les négociations pour l'élaboration du dispositif se sont élaborées entre les responsables de l'organisme de formation, les financeurs, le conseil régional qui structure la formation des formateurs au niveau régional, l'équipe d'enseignants-chercheurs de l'université et le consultant d'un cabinet chargé d'évaluer l'action et ses effets. Du côté de l'entreprise il s'agit d'un plan de formation, du côté de l'université il s'agit d'une formation universitaire diplômante.

12 L'idée initiale est la suivante : l'offre de formation prendra en compte l'expérience des formateurs et formatrices et devra les conduire à l'obtention de la licence. Les salariés 
seront volontaires pour s'engager dans ce parcours. Le temps de cette action se déroulera pour partie sur le temps de travail et pour partie sur le temps personnel et se déroulera sur une année universitaire.

Cette offre est donc à mi-chemin entre la VAE et un processus «classique » de formation pour obtenir la licence.

Alors que l'accès à l'expérience vécue est si difficile, de nombreux acteurs (sociaux, politiques, institutionnels, individuels) ont à cœur d'en faire valoir les acquis. Nous savons cependant que l'expérience n'est réductible ni à la performance réalisée dans l'exécution d'une tâche, ni au discours que l'auteur de la tâche peut tenir sur sa réalisation, l'une des difficultés parmi d'autres est liée au fait que l'auteur de l'activité la réduit bien souvent à la tâche réalisée (Clot, Diallo, 2003). Or cette expérience mobilise le vécu de la personne, les différents obstacles identifiés dans les recherches réalisées sur la VAE ont donc permis de poser les bases d'un dispositif qui permettrait de prendre en compte les acquis de l'expérience, sans prétendre y accéder réellement.

En effet, le dispositif de VAE, ouvert à toute personne active, constitue un moyen permettant notamment d'éviter d'avoir à réapprendre ce qu'elle sait déjà pour obtenir une certification. Cependant il présente de nombreuses difficultés car il s'agit de :

- produire un écrit impliqué et subjectif, manifestant l'autonomie, et engageant l'écrivant, ses valeurs et son rapport au travail,

- soumettre cet écrit à une grille d'évaluation hétéronormée, le référentiel d'activités et de compétences de la certification visée, grille qui est elle-même soumise à

l'interprétation subjective des évaluateurs,

- ne pouvoir faire évaluer que le produit fini, le processus par lequel les candidats ont acquis telle ou telle connaissance échappant à l'évaluateur voire au candidat,

- être en capacité de faire des choix pertinents d'expériences car toutes les expériences ne sont pas source de valeurs.

Pour réussir, les candidats à la VAE doivent donc être en capacité de s'engager seuls, se sentir compétents, en confiance, dégagés des affects et doivent savoir mobiliser leurs capacités d'expression écrite et orale au sein d'une situation d'évaluation. Les personnes non diplômées et concernées par le dispositif, objet de cette communication, ne sont assurément pas celles qui sont le plus en capacité de mobiliser l'ensemble de ces compétences.

17 Face à ces difficultés, il est donc convenu entre partenaires (universitaires, entreprise, des salariés concernés par la formation et financeurs) à l'origine du dispositif, destiné à accueillir des candidats ayant une expérience significative de formateurs d'adultes et non diplômés, que celui-ci articulera trois composantes :

1. l'analyse des pratiques conduisant à la reconnaissance des acquis issus des expériences,

2. les apports théoriques et méthodologiques liés à une analyse des besoins de formation identifiés tout au long du parcours,

3. l'écriture d'un mémoire "d'expérience » et son accompagnement, et une démarche qui consistera à :

- mettre en confiance,

- accompagner le candidat dans la démarche d'analyse des pratiques et d'écriture selon le principe de l'étayage (Vygotski, 1985). 
- dissocier l'écriture de l'évaluation. Ce n'est qu'en deuxième temps qu'il sera possible, par un processus d'auto évaluation assistée, de vérifier la conformité avec les attentes du référentiel et qu'il sera question d'évaluation,

- associer oral et écrit (Bakhtine, 1977), oral et écrit s'étayant et s'enrichissant mutuellement (Delamotte, 2000). traditionnelle. Comprendre le contexte synchronique et diachronique dans lequel il s'insère permet de saisir les enjeux sous-jacents à ce dispositif ainsi que les tensions historiques qui ne sont pas sans effets sur les attentes des différents partenaires.

\subsection{La prise en compte de l'expérience : contexte synchronique et diachronique}

L'expérience professionnelle étant au cœur de ce dispositif, il s'agit donc d'interroger dans un premier temps la place et le rôle que joue ou devrait jouer la prise en compte de l'expérience dans le contexte socio-économique actuel.

Lors du conseil européen de Lisbonne (2000), le savoir a été identifié comme étant la ressource principale du développement économique et social. Dans ce cadre s'est développée l'idée d'une professionnalisation durable: chaque personne doit être en capacité de renouveler ses savoirs et se requalifier au fur et à mesure des évolutions technologiques. La tendance à l'accroissement des aspects professionnalisants des études universitaires entre dans le cadre de la Formation Tout au Long de la Vie (FTLV).

En effet, le développement de la FTLV permettant à chacun de se qualifier et de se requalifier durant toute sa carrière constitue l'axe prioritaire de l'Europe pour atteindre 
un objectif: devenir la société de l'économie de la connaissance la plus compétitive du monde. Le livre blanc consacré à l'éducation et la formation de 1995 symbolise cette nouvelle direction stratégique de développement économique envisagée par l'Union Européenne.

Cette FTLV doit permettre à un plus grand nombre d'accéder à la connaissance. Cet accès du plus grand nombre à la connaissance n'est pas dénué d'enjeux. Selon Frétigné (2010), la période actuelle est traversée par une double interrogation source de tensions " [...] les inégalités d'accès à la formation enregistrées par les enquêtes nationales et européennes sont [...] vécues comme une injustice sociale mais surtout comme une source d'inefficacité économique ».

Cette volonté se trouve donc être en tension entre une conception libérale pour laquelle le droit individuel tend à rendre responsable chaque individu de son employabilité et de ses qualifications et une conception qui tendrait à considérer ce droit à la formation comme un signe d'équité permettant à ceux qui n'ont pas eu la chance préalable de se former d'entrer dans ce processus notamment par la prise en compte de l'apprentissage par expérience. Cette tension n'est pas nouvelle.

Si l'on remonte dans l'histoire, on constate dans les propos d'Aristote et plus encore chez ceux de Platon, que les individus étant considérés inégaux de fait, (mais n'est-ce pas le cas encore aujourd'hui ?) il fallait organiser leur sélection pour constituer les classes supérieures, la justice de cette époque consistant à faire coïncider les aptitudes et les positions sociales. En tant que rouages de cette division et particulièrement en France se trouvent la formation, la considération accordée à l'expérience professionnelle et les certifications.

Depuis le début du 20ème siècle la problématique de la reconnaissance de l'expérience professionnelle chez les adultes s'est posée de manière nouvelle. Les pouvoirs politiques ont décidé que les savoirs acquis par l'expérience seraient évalués par un examen et certifiés par un diplôme public (Brucy, 2007). Cette certification devait être systématiquement associée à une formation et le diplôme délivré "avait une valeur prédictive quant aux compétences du salarié au moment de l'embauche » (Brucy, 2007 p.19). S'opérait simultanément une distinction entre diplômés et non diplômés, le diplôme permettant d'accéder à une certaine place dans la division sociale du travail.

À cette époque la notion d'expérience n'était pas discutée, elle était mesurée à l'ancienneté. Considérée comme indispensable elle était cependant jugée insuffisante pour accéder au statut d'ouvrier complet. La formation était un incontournable de ce processus, celle-ci procédait d'un choix politique qui visait, au-delà du développement de la dimension économique du travailleur, le développement du citoyen et de l'homme.

Depuis lors cette formation a toujours été sous tendue par plusieurs enjeux : ceux de la promotion sociale, ceux de la gestion des ressources humaines identifiés, notamment, depuis la loi de 1971, et ceux de l'éducation permanente. Comme le dit Claude Dubar, si l'expression promotion sociale (Bonnet, 1999), définie comme changement de catégorie socioprofessionnelle, présidait au discours des années soixante, de préférence à éducation permanente, cette expression a connu des transformations associées aux transformations juridiques. Les lois de 1978, 1984, 1993, et plus récemment 2004 et 2009 dissocient les justifications économiques de la formation de son objectif social : la promotion sociale. La formation continue des adultes est devenue une obligation et, actuellement, on peut « interpréter les mouvements de la formation continue comme des remodelages des mécanismes de reproduction sociale» (Dubar, 1999, p 48). Les effets produits de la 
formation des adultes ne sont pas ceux attendus. Loin de corriger les inégalités sociales ce système les a accentuées (Presse, 2008, p29). Cependant l'une des orientations de la stratégie de Lisbonne est de remédier à ces effets et donc d'engager les moins qualifiés à s'impliquer dans un processus de formation et de certification, et comme cela vient d'être énoncé, non seulement pour leur promotion mais pour progresser vers l'économie de la connaissance. L'obtention d'une certification est devenue incontournable, celle-ci jouant entre autre la fonction de passeport pour l'emploi et ce quel que soit le mode d'acquisition, avec ou sans formation.

Actuellement les différentes approches des organisations porteuses du projet de FTLV traduisent les mêmes interrogations : la formation au service de quels enjeux ? D'un côté, l'approche humaniste (UNESCO) valorise la promotion sociale des personnes, de l'autre côté l'approche économique (OCDE) se place en faveur de la connaissance comme moyen économique de développement de la société. On retrouve dans cette dernière la théorie du capital humain que l'on pourrait nommer plus précisément «théorie du capital expérientiel collectif» (Presse, 2007). Il est ainsi énoncé qu' « une plus grande visibilité des savoirs, savoir-faire et/ou compétences, indépendamment de la manière dont ils ont été acquis, pourrait fluidifier le marché du travail et le rendre plus efficace " (Werquin, 2010, p. 17). Voici donc affiché l'un des intérêts de la prise en compte de l'expérience: rendre visible tous les savoirs. Les incidences sur les formations universitaires sont directes.

Le dispositif mis en place se situe donc bien au cœur de ces tensions ayant, d'une part, toujours existées entre expérience et formation au risque de nier les aspects formatifs de la formation autres que ceux de l'acquisition de savoirs formels, tel que l'esprit critique par exemple, et d'autre part entre développement économique et développement des personnes.

\subsection{Garantir la certification}

Ce dispositif est encadré par un discours européen qui renforce cette nécessité de permettre l'accès à la certification de tous les travailleurs. En effet, dans le cadre du nouveau cycle de la stratégie de Lisbonne (2008-2010) «il importe de renforcer le soutien apporté aux travailleurs peu qualifiés, aux migrants et aux handicapés, notamment en encourageant le développement de leurs compétences » (commission conseil européen, 2007, p. 11).

Le renforcement du pôle éducation du triangle de la connaissance (p. 10) formé par la recherche, l'innovation et l'éducation est préconisé car il est essentiel d'investir dans le capital humain pour progresser vers l'économie de la connaissance, voire de combattre les inégalités. Pourquoi inciter ces travailleurs faiblement qualifiés à se former?

Cette orientation est assortie du développement des dispositifs professionnalisants au sein de l'université. Ceux-ci s'imposent un peu comme "un allant de soi». Cette place accordée à ces processus de professionnalisation invite cependant à les situer par rapport à la formation et à l'enseignement. Les trois modalités, enseignement, formation, professionnalisation, ont des implications identitaires différentes car elles entretiennent des rapports différents avec l'environnement (Barbier, 2006). Dans le cadre de l'enseignement, les rapports sont essentiellement pensés en termes de conceptualisation et la transformation identitaire se produit par un processus d'assimilation appropriation. Le cadre de la formation conduit à entretenir une relation dialogique entre action et 
réflexion, il s'agit d'un processus de décontextualisation-recontextualisation qui favorise l'émergence de nouvelles pratiques. Le processus de professionnalisation, dont la référence centrale est la compétence, est fondé sur une logique de réflexion sur et pour l'action préparant directement à l'exercice du travail (Wittorski, 2007). Ces trois processus peuvent être mis en œuvre au sein de l'université, le processus de professionnalisation est cependant le plus récent et il occupe une place importante dans les dispositifs de formation universitaire. La nécessité d'effectuer des stages à tous les niveaux de formation, le développement des dispositifs professionnels et la prise en compte du taux d'insertion professionnel des étudiants dans l'évaluation des universités en sont les marques.

Il apparaît donc que, dans ce contexte européen, la formation professionnalisante des travailleurs faiblement « qualifiés » et l'obtention, par ceux-ci, d'une certification soient une priorité qui vise deux enjeux: progresser vers l'économie de la connaissance et réduire les inégalités sociales.

Cette priorité est assortie, en France, de dispositions juridiques visant à faciliter l'accès à la qualification, notamment à travers d'une part la mise en place des dispositifs de Validation des Acquis de l'Expérience et d'autre part par le développement des Droits d'accès Individuels à la Formation (DIF). Ces deux dispositions sont au cœur de l'offre de formation élaborée pour permettre à des formateurs d'adultes non « qualifiés » d'obtenir une certification universitaire.

38 L'analyse du contexte a fait apparaître les tensions sous-jacentes historiques et socioéconomiques, les textes européens constituent les discours qui encadrent ce dispositif, discours qui prône la professionnalisation, plutôt que la formation, discours qui sont assortis, en France, de dispositifs juridiques pour la mise en œuvre.

\subsection{Prendre appui sur les dispositifs juridiques}

Les négociations, comme énoncées ci-dessus, sont déroulées entre les responsables de l'organisme de formation, les financeurs (OPCA), le conseil régional qui structure la formation des formateurs au niveau régional, l'équipe d'enseignants-chercheurs.

Dans ce cadre et afin de légitimer la démarche un organisme extérieur est sollicité pour évaluer « l'expérimentation ", et ce dans un but de généralisation de l'offre de formation.

41 Du côté de l'entreprise il s'agit d'un plan de formation au sein duquel le DIF constitue l'une des pièces importantes du financement de l'action. Les salariés signent un contrat d'engagement vis-à-vis de l'entreprise assurant à terme, pour l'entreprise, le réinvestissement de l'action dans son développement. Obligation est faite pour les salariés d'élaborer un portefeuille de compétences. Un modèle à entête de l'entreprise est proposé.

42 Lors de l'adoption de la loi du 4 mai 2004 relative à "la formation professionnelle tout au long de la vie et au dialogue social" des désaccords régnaient sur le sens accordé au droit individuel à la formation (DIF) et plus précisément sur la répartition des responsabilités entre employeurs et salariés, sur les modalités de mise en œuvre de ce droit et sur ses finalités. Les points de vue de Merle et de Lichtenberger (cités par Frétigné) expriment cette tension: d'une part «le droit individuel [...] doit permettre aux entreprises de gérer les compétences. [...] Ce droit prend principalement la forme d'une incitation à l'épargne individuelle: chacun est incité, par exemple à travers les avantages fiscaux, à contribuer à sa propre formation 
. (Frétigné, p. 82)»; d'autre part ce droit individuel à la formation doit être considéré comme un bien collectif octroyé sous un principe d'équité et comme un investissement bénéfique à l'individu mais surtout à la collectivité en terme d'élévation des niveaux de formations devant profiter à l'ensemble de la société. Ce DIF constitue l'une des sources de financement de l'action de formation cependant l'engagement des personnes n'est pas "volontaire " pour tous. On se trouve ici dans une situation d'incitation de l'entreprise à s'impliquer dans ce projet de montée en qualification.

Les dispositions politiques économiques et juridiques encadrent tous les partenaires chargés de la mise en œuvre des dispositifs. À leur niveau, ceux-ci font valoir leur point de vue et défendent leurs intérêts immédiats et ou perceptibles.

\section{Le dit et le non-dit : Les discours des partenaires}

Cette idée d'élaborer une offre de formation est née à la suite d'une communication publique sur les possibilités d'usage de la VAE au sein de dispositifs de formations universitaires ${ }^{1}$.

Comme toute négociation celle-ci met en jeu des groupes d'acteurs (entreprise, université, salariés, partenaires financiers) aux intérêts différents qui se réunissent cependant autour d'un objectif commun: la mise en place d'un dispositif de formation prenant en compte l'expérience professionnelle. Les attentes des différents partenaires n'ont pas toutes été explicitées, certaines sont apparues implicitement au fil du déroulement et manifestent des intérêts divergents.

\subsection{Les discours et les attentes de l'entreprise}

Soumis aux lois concurrentielles du marché de la Formation des adultes et aux financements possibles pour ses propres salariés, les responsables de l'organisme de formation au sein duquel travaillent les formateurs attendent une implication de ses salariés et un retour sur investissements. Ils attendent de l'université une professionnalisation de ses salariés et une montée en qualification professionnelle. Pour cela ils libèrent du temps de travail afin de rendre possible le suivi de la formation mais demandent un co-investissement de la part de ceux-ci. Les attentes énoncées de l'organisme sont les suivantes : sécuriser les parcours, valoriser l'expérience, qualifier les professionnels, se situer sur le marché concurrentiel. Le discours conjugue simultanément une volonté de promotion sociale et professionnelle pour les salariés et une volonté de conserver une place dans le monde économique.

Ce dispositif est donc davantage porté par les responsables de l'OF et non par ses salariés. Il a pour le but de mobiliser les salariés, d'améliorer leurs performances, de renforcer leur sens des responsabilités, d'augmenter leur efficacité.

\subsection{Le discours et les enjeux des financeurs et des politiques}

Les financeurs (non cités par respect de l'anonymat) acceptent de prendre en charge les financements sous réserve que les salariés mettent à disposition de ce projet leur Droit Individuel de Formation, qu'ils signent un contrat les engageant à poursuivre dans l'entreprise et qu'ils élaborent un portefeuille de compétences afin de les responsabiliser 
dans leur employabilité. L'intérêt du dispositif est pour partie lié à la réduction du temps de formation, à la réduction du coût et à la montée en certification des seniors, source potentielle de marchés financiers. Il intéresse les politiques comme nouvelle forme de formation possible.

\subsection{Le discours et enjeux du côté de l'université} (es financeurs ne visent pas les mêmes objectifs. Ce dispositif vise une sécurisation des parcours par une montée en qualification et une reconnaissance des expériences, la volonté de promotion professionnelle est partagée par l'université et l'organisme mais en arrière fond et bien que non énoncée au départ de la négociation, il y a nécessité pour l'organisme que les professionnels obtiennent la licence pour répondre aux appels d'offre, source de financement de l'organisme. Cette montée en qualification attendue répond prioritairement à une gestion des compétences. Ces tensions et contradictions se sont particulièrement manifestées au sein de deux composantes du dispositif: l'une relative à l'élaboration d'un portefeuille de compétences, l'autre relative à l'espace de formation.

\subsection{Du portefeuille de compétences au portfolio} dispositif, l'idée d'un portefeuille de compétences n'a pas suivi exactement le chemin prévu.

L'idée d'un portfolio est venue contre balancer la proposition du portefeuille (le portefeuille est davantage un outil lié à la gestion des compétences par l'entreprise, le portfolio un récapitulatif personnel : une distinction subtile).

Les salariés de l'organisme souhaitent obtenir le diplôme, certains se sont engagés volontiers, d'autres moins confiants l'ont fait avec réserve, d'autres enfin l'ont vécu comme un défi à relever. Mais tous ont interrogés les composantes du dispositif dont la 
nécessité du portefeuille comme l'indiquent ces propos extraits du rapport d'évaluation opéré par un extérieur : «les stagiaires n'ont pas compris le besoin de portefeuille de compétences qui émanait de l'entreprise et non de l'université ». Ils ont éprouvé une certaine méfiance à l'égard de ce document à élaborer, qui leur semblait manifester un certain contrôle de l'entreprise sur eux-mêmes.

Afin de dépasser cette contrainte celle-ci a été transformée: faire du portefeuille de compétences un portfolio personnalisé (Layec, 2006). Le modèle de portefeuille proposé par l'entreprise était assorti du logo de l'entreprise, outil ayant une fonction de passeport dans les transactions au sein du marché du travail et permettant de répondre à la demande de traçabilité de l'expérience. Le glissement opéré vers le portfolio a permis à chacun des stagiaires de s'approprier la démarche à son envie, retenant les expériences significatives pour lui, les enjeux ayant été clairement explicités: il s'agit d'un outil personnel qui permet d'élaborer en fonction des besoins (personnels, professionnels ou autres) d'autres outils, dont le portefeuille de compétence, pouvant être mis à disposition d'autrui.

\subsection{Espace de formation, espace de travail}

Les tensions les plus manifestes se sont déroulées dans le rapport à l'espace. Elles se sont structurées autour de trois dimensions : le lieu de formation, la place de la formation, la distribution symbolique des diplômes.

La formation s'est déroulée, comme convenu initialement, dans l'entreprise. Au démarrage cela semblait être un atout pour les stagiaires, l'entreprise étant un lieu sécurisant au sein duquel ils allaient affronter "les savoirs universitaires et les enseignants » et qui mettait ces derniers à distance du monde universitaire. D'une certaine manière, la formation proposée sortait de la « forme scolaire traditionnelle et de ses modes de socialisation imposés » (Vincent, 1994).

Mais cette disposition privilégiait l'espace de travail, avec également ses contraintes et ses modes de socialisation: suivre une formation dans son entreprise rendait les stagiaires disponibles pour toutes autres activités, en fonction des besoins et des priorités de l'entreprise. La formation répondait donc à un besoin parmi d'autres, ne permettant peut être pas d'offrir aux stagiaires la distance critique et la prise de recul nécessaire. C'est à ce niveau que se trouve la manifestation la plus visible du risque d'instrumentalisation de la formation, risque qui s'est concrétisé dans une première distribution symbolique des diplômes, en présence des financeurs et politiques et ce à l'initiative de ces derniers et hors de l'université.

Derrière ce rapport à l'espace et à sa dimension symbolique ne retrouve-ton pas là le débat du début du XXème siècle dans les démarches préalables à la mise en place du CAP ou la différence essentielle entre la formation entreprise et la formation institutionnelle résidait dans l'acquisition de connaissances nécessaires à la citoyenneté, tensions toujours présentes (Cahuc, Kramarz, 2005)?

\section{En conclusion et discussion}

60 Pour conclure tentons de répondre à la question initiale «quelles tensions et contradictions se sont manifestées dans la mise en place d'un dispositif universitaire 
s'appuyant sur l'expérience co-élaboré par les financeurs et politiques, l'entreprise concernée par les salariés-stagiaires et l'université ?».

Il n'était pas de notre propos de tenter de répondre à la question de l'accès à l'expérience mais bien d'essayer de cerner les tensions et les contradictions liées aux usages de l'expérience dans un dispositif de formation universitaire.

Différentes questions se posaient: les attentes de l'entreprise n'étaient-elles pas de valoriser le capital expérientiel collectif pour le mettre au profit de l'entreprise, capital qui devrait correspondre aux attentes de l'entreprise, au risque de desservir ceux qui souhaiteraient valoriser des expériences qui ne correspondraient pas à ces attentes? N'y avait-t-il pas risque d'un renforcement de contrôle du monde économique sur la distribution des diplômes et par la même un retour en arrière valorisant l'apprentissage sur le tas? À quelles conditions l'université pourrait-elle poursuivre sa démarche innovante en vue de favoriser la promotion des personnes non qualifiées?

Pendant le parcours ces questions se sont renforcées: les attentes de l'entreprise en matière de capacités à répondre aux appels d'offre se sont faites explicites. La réalisation du portefeuille de compétence a été présentée, en milieu de parcours, comme un incontournable du plan de formation et un engagement de l'entreprise dans le cadre du financement.

64 La co élaboration de l'ensemble de la démarche par les différents partenaires a conduit à appropriation de l'espace de formation par l'entreprise et les partenaires, cette appropriation allant jusqu'à la prise en charge de la « délivrance ».

Comme on le voit ci-dessus le dispositif a toujours été en tension entre des intentions de professionnalisation de l'entreprise appuyée par les politiques régionales et des intentions de promotion sociale. Mais le rapport expérience formation est depuis longtemps en tension, on peut donc se demander ce qu'il y a de nouveau. Il semblerait que c'est autour de la notion de professionnalisation que se cristallisent les nouvelles tensions et la généralisation d'un tel dispositif conduit, préalablement, à d'autres questions. Le projet des organismes de formation est de "professionnaliser ses professionnels » en allant chercher ce qui correspond à des besoins que ces organismes auraient identifiés. Dans le dispositif cité ci-dessus, les tensions se sont manifestées, notamment autour de l'espace de formation laissant apparaitre des risques d'instrumentalisation de la formation. Derrière cette professionnalisation, se déroulant sur le terrain professionnel, il y a donc bien assurément la nécessité de faire valoir les expériences acquises, mais se pose alors une question : «cela n'accroît-il pas le risque de n'accorder de valeur qu'aux compétences et expériences strictement utiles au travail ?»

\section{BIBLIOGRAPHIE}

Bakhtine, M. (1977). Le Marxisme et la philosophie du langage : Essai d'application de la méthode sociologique en linguistique. Paris : Editions de Minuit. 
Barbier, J.M. (1996). De l'usage de la notion d'identité en recherche, notamment dans le domaine de la formation, Éducation permanente, 128, pp11-26

Barbier, J.M. (2006). Les voies nouvelles de la professionnalisation. In Yves Lenoir, Marie-Hélène Bouillier-Dudot (éd.), Savoirs professionnels et curriculum de formation. (pp 67-82). Saint Nicolas (Quebec), Presses universitaires de Laval.

Bonnet. B. (1999). La formation professionnelle des adultes. Une institution et ses formateurs. Paris :

L'Harmattan.

Bourgeois, E. (1997). Apprentissage et formation d'adultes. Paris : PUF.

Boussard, V. et al. (2010). L'injonction au professionnalisme : analyse d'une dynamique plurielle. Rennes, PUR.

Brucy, G. ( 2007). La certification quelques points d'histoire, Relief, 20, 19-32.

Cahuc,P., \& Kramarz, F. (2005). De la précarité à la mobilité : vers une sécurité sociale professionnelle. Paris : La documentation française.

Clot, Y., \& Diallo, M.D. ( 2003). L'exploration de l'expérience dans l'analyse de l'activité : Problème de méthode, Orientation Scolaire et Professionnelle, 32(2), 203-217.

Commission européenne. (1995). Livre blanc sur l'éducation et la formation : enseigner et apprendre - vers la société cognitive. [en ligne] disponible le 1 mars 2012 sur http:// www.teipat.gr/pages/stud_exchange/leonardo/introf.html

Conseil européen de Bruxelles, (mars 2008). Conclusion de la présidence. [en ligne] disponible le 1 mars 2012 sur http://www.consilium.europa.eu/ueDocs/cms_Data/docs/pressData/fr/ ec/99435.pdf

Conseil Européen de Lisbonne [en ligne] disponible le 1 mars sur http:// www.europarl.europa.eu/ftu/pdf/fr/FTU_4.1.pdf

Communication de la Commission au Conseil européen - Rapport stratégique concernant la stratégie renouvelée de Lisbonne pour la croissance et l'emploi : lancement du nouveau cycle (2008-2010) - Garder la cadence des réformes - Évaluation des programmes nationaux de réforme /* COM/2007/0803 final. (2007) [en ligne] disponible le 1 août 2012 sur http://eur-lex.europa.eu/ LexUriServ/LexUriServ.do?uri=CELEX:52007DC0803(02):FR:HTML

Delamotte, R. et al (2000). Passage à l'écriture : un défi pour les apprenants et les formateurs. Paris : PUF.

Dubar, C. (1999). De la deuxième chance au co-investissement, brève histoire de la promotion sociale (1959-1993), In Dubar C. \& Gadea C. (ED.), La promotion sociale en France, (pp. 31-49). Lille : Septentrion.

Frétigné C. (2010). Ce que "life learning" veut dire en France, Actualité de la formation permanente, 224/225, 80-86.

Foucault, M. (2001). (1970-1975), Le jeu de Michel Foucault, in Dits et Ecrits, T.II. (1970-1975), éd. Gallimard, Paris.

Kaddouri, M. (1999). Engagement en formation et dynamique identitaire, Recherche et Formation, 31, 101-112.

Kergoat, P. (2010). Les formations par apprentissage : un outil au service d'une démocratisation de l'enseignement supérieur? Net.doc, 75.

Layec, J. ( 2006). Auto-orientation tout au long de la vie : le portfolio réflexif. Paris : L’Harmattan. 
Presse, M.C. (2007). La validation des acquis de l'expérience : mythes et imaginaires sociaux . In Jorro, A. (éd.), Evaluation et développement professionnel (pp 203-214). Paris, L’Harmattan.

Presse, M.C. (2008). La validation des acquis de l'expérience : une nouvelle forme d'exclusion, Contradictions, 123.

Presse, M.C. (2011). De la validation des acquis de l'expérience à la professionnalisation des formateurs, Education permanente, 188, 37-52.

Vincent, G. (1994). L'éducation prisonnière de la forme scolaire ? Scolarisation et socialisation dans les sociétés industrielles. Lyon : PUL.

Vygotski, L.S. (1985).Pensée et langage. Paris : Messidor : Editions sociales.

Werquin, P. (2010). Reconnaître l'apprentissage non formel et informel : Résultats, politiques, pratiques. Paris : OCDE.

Wittorski, R. (1997). Analyse du travail et production de compétences collectives. Paris : L'Harmattan.

\section{NOTES}

1. A l'initiative de cette rencontre se trouvent les acteurs régionaux ayant en charge la formation des formateurs.

\section{RÉSUMÉS}

La valorisation et la reconnaissance de l'expérience se développent dans le monde sous différentes formes ouvrant, notamment en France, une nouvelle voie d'accès pour l'obtention d'une certification. Les différents travaux de recherche effectués sur la VAE depuis 2002 (Presse, 2008) ont permis de mettre en place un dispositif de formation, à l'université. Les enjeux et les effets sont les objets de l'analyse présentée. Il ne s'agit pas de fournir des éléments de réponse sur les possibilités d'accès à l'expérience, mais de comprendre certaines tensions que son usage crée. Il apparait que c'est dans le rapport à l'espace que se situent les plus fortes tensions.

Experience recognition and its different forms around the world open particularly in France, a new access pathway to a certification. The various research on the APL (Accreditation of Prior Learning) or in French,VAE (Validation des Acquis de l'Expérience) since 2002 (Press, 2008) have helped to set up a training program at the university. Issues and effects of this device are the objects of the analysis. The matter is not therefore to provide some answers on access opportunities to the experience, but to understand the tensions that its use creates. It appears that the greatest tensions have to be seen in relation to the training space.

\section{INDEX}

Mots-clés : expérience, certification, contradictions, formation d'adultes

Keywords : adult éducation 
AUTEUR

MARIE-CHRISTINE PRESSE

Professeure en sciences de l'éducation, Université de Lille 1, Institut CUEEP, Membre de CIREL équipe Trigone 